\title{
Zur Kaskadentheorie der Trennung polynärer Isotopengemische
}

\author{
Alfred Narten \\ Chemistry Division, Oak Ridge National Laboratory *, Oak Ridge, Tennessee \\ (Z. Naturforschg. 20 a, 663-666 [1965]; eingegangen am 14. Februar 1965)
}

\begin{abstract}
The theory of isotope separation in cascades is extended to polynary mixtures. Conditions for minimum reflux (maximum production) are derived. For square cascades, the general solution of the differential equations is indicated; a special solution is given for the important case of large reflux ratio. The enrichment of middle components in ternary mixtures is discussed, it is shown to be impractical in a single square cascade.
\end{abstract}

\section{Einzeltrenneffekt und Trennelement}

In einem Gemisch aus $n$ Komponenten ist der Einzeltrenneffekt einer hervorgehobenen Spezies $k$ gegenüber einer beliebigen Bezugskomponente $j$ definiert durch den Trennfaktor

$$
\alpha_{k}=\frac{N_{k} / N_{j}}{N_{k^{\prime}} / N_{j}^{\prime}}>1 \text {. }
$$

$N$ und $N^{\prime}$ sind die relativen Konzentrationen zweier Phasen im thermodynamischen Gleichgewicht. Die Begriffe Phase und Gleichgewicht seien so umfassend definiert, daß verschiedenartige Trenneffekte, wie Dampfdruckunterschiede, Unterschiede in der Gleichgewichtskonstanten chemischer Reaktionen, Diffusionseffekte etc., in die Betrachtung einbezogen sind ${ }^{1}$. In Gemischen isotoper Verbindungen ist der Trennfaktor $\alpha_{k}$ in den meisten Fällen von 1 nur wenig verschieden, so daß es vorteilhaft ist, mit dem Trennparameter $\varepsilon_{k}$ zu rechnen,

$$
\alpha_{k}-1=\varepsilon_{k} \ll 1 .
$$

Es ist für das Folgende zweckmäßig, ein Trennelement (Abb. 1) zu definieren; die Phasenströme $L$ und $l$ seien nach Durchströmen dieses Trenn-

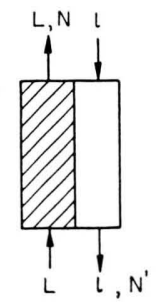

Abb. 1. Trennelement.

\footnotetext{
* Research sponsored by the U.S. Atomic Energy Commission under contract with the Union Carbide Corporation.

1 H. London, Separation of Isotopes, George Newnes Ltd., London 1961, p. 9.
}

elementes im Gleichgewicht (1). Ein solches Trennelement kann eine diskrete Einheit sein, wie z. B. eine konvektionslose Zentrifuge; es kann auch eine fiktive Einheit sein, wie die Trennstufenhöhe (HETP) einer Gegenstromkolonne.

\section{Trennkaskaden}

Zur effektiven Trennung von Isotopengemischen ist es notwendig, einzelne Trennelemente so zu kombinieren, daß der Einzeleffekt vervielfältigt wird ${ }^{2}$. Eine beliebige Anordnung von Trennelementen zu einer Trennkaskade ist in Abb. 2 schematisch dargestellt.

Die Verteilung der $n$ Gemischkomponenten entlang der $z$-Koordinate einer Trennkaskade wird in üblicher Weise ${ }^{3}$ aus Stoffbilanz- und Gleichgewichtsbetrachtungen abgeleitet. Für den stationären $\mathrm{Zu}$ stand erhält man ein System von $(n-1)$ Differentialgleichungen von der Form

$$
\frac{\mathrm{d} N_{k}}{\mathrm{~d} \varepsilon_{k} s}=N_{k}\left(1-\sum_{i} K_{i} N_{i}\right)-\psi_{k}\left(N_{p}^{P}-N_{k}\right) .
$$

Bei der Ableitung von (3) wurden die Werte für den Durchsatz $L$ und die Entnahme $P$ so gewählt, daß die hervorgehobene Komponente $k$ hinsichtlich der Bezugsspezies $j$ in der positiven $z$-Richtung angereichert wird (Abb. 2). $s$ ist die Anzahl Trennelemente (Trennstufen) entlang der $z$-Koordinate der Kaskade; $\psi_{k}=P / L \varepsilon_{k}$ ist der Entnahmeparameter, welcher die Verteilung der $k$-ten Komponente entlang der Kaskade bestimmt; $K_{i}=\varepsilon_{i} / \varepsilon_{k}$ sind die auf das betrachtete Komponentenpaar $k$ und $j$ nor-

2 W. Kunn, Dechema-Monographien, Bd. 21 [1952], p. 238 bis 257.

${ }^{3}$ K. Cohen, The Theory of Isotope Separation, National Nuclear Energy Series, III-1B, McGraw-Hill Book Co. Inc., New York 1951. 
malisierten Trennparameter der übrigen Gemischkomponenten. Im Falle von Isotopengemischen sind die $K_{i}$ kleine ganze Zahlen.

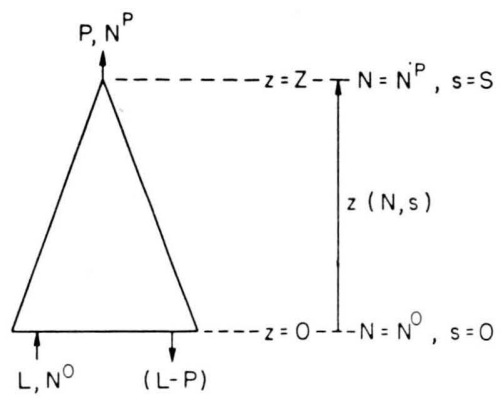

Abb. 2. Trennkaskade.

Die rechte Seite von (3) und somit $\mathrm{d} N_{k} / \mathrm{d} \varepsilon_{k} s$ nimmt mit wachsendem $N_{k}$ zu. $\mathrm{d} N_{k} / \mathrm{d} \varepsilon_{k} s$ nimmt den kleinsten Wert an der Basis der Kaskade an $(z=0$ in Abb. 2). Der maximal mögliche Transport wird realisiert, wenn

$$
\left.\frac{\mathrm{d} N_{k}}{\mathrm{~d} \varepsilon_{k} s}\right|_{z=0}=0 .
$$

Aus (3) und (4) ergibt sich für die maximale Entnahme an Komponente $k$ am Kopf der Kaskade $(z=Z, s=S$ in Abb. 2)

$$
P<\frac{L^{0} N_{k^{0}} \varepsilon_{k}\left(1-\sum_{i} K_{i} N_{i}^{0}\right)}{\left(N_{k} P-N_{k}\right)} .
$$

Gl. (5) gibt die maximale Entnahme $P$ für eine beliebige Kaskade als Funktion der Trennparameter, des Durchsatzes $L^{0}$ an der Basis der Kaskade, der Entnahmekonzentration $N_{k}{ }^{P}$ der $k$-ten Komponente am Kopf der Kaskade und der Ausgangskonzentrationen $N^{\mathbf{0}}$ aller Komponenten an.

Zur Kenntnis der Verteilung der $n$ Komponenten eines Isotopengemisches entlang der $z$-Achse (Abb. 2) einer Trennkaskade ist es notwendig, das System (3) zu integrieren. Dies ist in allgemeiner Form nicht möglich, da der Durchsatz $L$ als Funktion der $z$-Koordinate von der Form der jeweiligen Kaskade in empfindlicher Weise abhängt.

Lösungsmethoden des Systems (3) für verschiedene, praktisch interessante, gestaffelte Kaskaden sind für den polynären Fall beschrieben worden ${ }^{4}$.

\footnotetext{
4 A. De La Garza, G. A. Garret u. J. E. Murphy, Chem. Eng. Sci. 15, 188 [1961].

5 W. Kunn, Helv. Chim. Acta 29, 329 [1946].
}

\section{Kaskaden mit konstantem Durchsatz (Gegenstromkolonnen)}

Das Problem der polynären Isotopentrennung in Kaskaden mit konstantem Durchsatz (square cascades) ist bislang nur für die Sonderfälle $P=0^{5}$ und $n=2{ }^{3}$ gelöst worden. Eine solche Kaskade, als deren praktisch wichtigster Fall sämtliche Gegenstromkolonnen zu nennen sind, ist in Abb. 3 schematisch dargestellt.

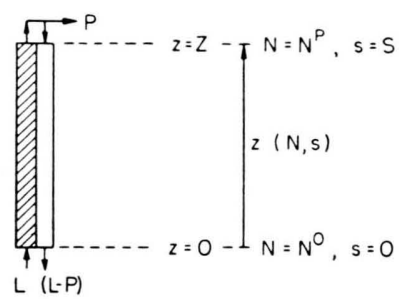

Abb. 3. Trennkaskade mit konstantem Durchsatz $L$ (square cascade) .

Ein einfacher, praktisch wichtiger Weg zur Integration des Systems (3) liegt in der Einführung eines mittleren Trennparameters $\bar{\varepsilon}$

$$
\bar{\varepsilon}_{k}=\frac{\varepsilon_{k}-\sum_{i} \varepsilon_{i} N_{i}}{\left(1-N_{k}\right)} .
$$

Einsetzen von (6) in (3) führt das System von $(n-1)$ Differentialgleichungen über in $(n-1)$ unabhängige Gleichungen formal binären Types:

$$
\frac{\mathrm{d} N_{k}}{\mathrm{~d} \varepsilon_{k} s}=N_{k}\left(1-N_{k}\right)-\bar{\psi}_{k}\left(N_{k}^{P}-N_{k}\right)
$$

mit $\bar{\psi}_{k}=P / L \bar{\varepsilon}_{k}$. In vielen praktisch interessierenden Fällen ( $\varepsilon s$ nicht zu groß) können die Komponenten $k$ und $j$ so gewählt werden, daß der mittlere Trennparameter längs der Kaskade konstant ist. Integration ergibt dann ${ }^{3}$ :

$s \bar{\varepsilon}=2 / \Delta\left(\bar{\psi}_{k}\right)$

$$
\begin{gathered}
\cdot \tanh ^{-1} \frac{\left(N_{k}-N_{k}^{0}\right) \Delta\left(\bar{\psi}_{k}\right)}{\left(N_{k}+N_{k}^{0}\right)\left(1+\bar{\psi}_{k}\right)-2 N_{k} N_{k}^{0}-2 N_{k}^{P} \bar{\psi}_{k}} \\
\text { mit } \Delta\left(\bar{\psi}_{k}\right)=\left[1+2 \bar{\psi}_{k}\left(1-2 N_{k}^{P}\right)+\bar{\psi}_{k}^{2}\right]^{1 / 2} .
\end{gathered}
$$

Das System (3) ist nichtlinear und nichthomogen; Substitution von $N_{i}=x_{i} / x_{v}$ mit $x_{v}{ }^{\prime}=\sum_{i} K_{i} x_{i}$ führt zu dem linearen System

$$
x_{k}{ }^{\prime}=\left(1+\psi_{k}\right) x_{k}-\psi_{k} N_{k}{ }^{P} x_{\nu}, \quad x_{\nu}{ }^{\prime}=\sum_{i} K_{i} x_{i},
$$

dessen allgemeine Lösung nach Rücksubstitution ist:

$$
\frac{N_{k} P}{N_{k}}=\frac{\sum_{i}\left(1+\psi_{k}+\lambda_{i}\right) C_{k i} \exp \left(\lambda_{i} \varepsilon_{k} s\right)}{\psi_{k} \sum_{i} C_{k i} \exp \left(\lambda_{i} \varepsilon_{k} s\right)}
$$


Die Konstanten $C_{k i}$ sind durch das System ( 3 a) und die Randbedingung $s=0, N=N^{0}$ bestimmt; $\lambda_{i}$ sind die Wurzeln des charakteristischen Polynoms

$$
\begin{array}{ccccc}
{\left[\left(1+\psi_{1}\right)-\lambda\right]} & 0 & \ldots & 0 & -\psi_{1} N_{1} P \\
0 & {\left[\left(1+\psi_{2}\right)-\lambda\right]} & \cdots & 0 & -\psi_{2} N_{2} P \\
\cdots & \cdots & \cdots & \cdots & {\left[\left(1+\psi_{n}\right)-\lambda\right]} \\
0 & 0 & \cdots & \psi_{n} N_{n} P \\
K_{1} & K_{2} & \cdots & K_{n} & -\lambda
\end{array}
$$

Die numerische Auswertung der allgemeinen Lösung (9) des Problems (3) ist so umständlich, daß es in den meisten praktischen Fällen zweckmäßiger sein dürfte, das Differentialgleichungssystem (3) direkt mit Hilfe elektronischer Rechenanlagen iterativ zu lösen. Das System (9) von $(n-1)$ transzen- denten Gleichungen ist jedoch von Bedeutung zur Gewinnung handlicherer Ausdrücke mit beschränkter Geltung.

Für den Fall $\psi_{i} \ll 1$ sind z. B. alle $\lambda_{i}$ reell, und (9) nimmt die einfache Form

$$
\varepsilon_{k} s\left[1+\psi_{k}\left(N_{j}^{P}-N_{k}^{P}\right)\right]=\ln \frac{N_{k} / N_{j}}{N_{k^{0}} / N_{j}^{0}}, \quad \psi_{k} \ll 1
$$

an. Die Verteilung der $n$ Komponenten entlang der Kolonne läßt sich explizit als Funktion der Entnahmeparameter, der Ausgangs- und Endkonzentrationen angeben:

$$
N_{k}=N_{k}^{0}\left\{\sum_{i} N_{i}^{0} \exp \left[s \varepsilon_{k}\left(K_{i}-1+\psi_{k}\left(N_{k}^{P}-N_{i}^{P}\right)\right)\right]\right\}^{-1}, \quad \psi_{k} \ll 1 .
$$

Für $\psi=0$ und $N=N^{P}$ gehen (10) und (10 a) in die bekannten einfachen Beziehungen ${ }^{6}$ über.

\section{Anreicherung von Mittelkomponenten}

Die Anreicherung mittlerer Komponenten polynärer Isotopengemische zählt zu den schwierigsten Trennproblemen überhaupt. Als Beispiele seien ${ }^{17} \mathrm{O}$, ${ }^{21} \mathrm{Ne},{ }^{29} \mathrm{Si}$ und ${ }^{33} \mathrm{~S}$ in den natürlich vorkommenden Gemischen, sowie ${ }^{236} \mathrm{U}$ in technisch anfallenden Reaktorbrennelementen genannt.

Die Sauerstoff-17-Anreicherung als typisches Mittelkomponentenproblem ist für den Fall der Wasserdestillation ${ }^{7}$ und der Thermodiffusion ${ }^{8}$ von Sauerstoffgas diskutiert worden. Gl. (5) zeigt, daß zur Erzielung nennenswerter ${ }^{17} \mathrm{O}$-Anreicherungen die Bedingung $\psi\left({ }^{17} \mathrm{O}\right)=P / L \varepsilon\left({ }^{17} \mathrm{O}\right) \ll 1$ notwendig ist. In Abb. 4 ist $N^{P}\left({ }^{17} \mathrm{O}\right)$, die Sauerstoff-17-Konzentration am Kopf einer beliebigen Gegenstromkolonne (Abb. 3) als Funktion des Entnahmeparameters $\psi\left({ }^{17} \mathrm{O}\right)$ für verschiedene Kolonnenlängen $S \varepsilon\left({ }^{17} \mathrm{O}\right)$ nach Gl. (10) aufgetragen $\left(N=N^{P}\right)$. Die Konzentrationen an der Basis der Kaskade sind die des natürlichen Isotopengemisches:

$$
99,759 \%{ }^{16} \mathrm{O}, \quad 0,037 \%{ }^{17} \mathrm{O}, \quad 0,204 \%{ }^{18} \mathrm{O} .
$$

Wie Abb. 4 zeigt, ist die Anreicherung von ${ }^{17} \mathrm{O}$ in einer Gegenstromkolonne mit Entnahme am Kopf der Kaskade praktisch auf Konzentrationen von weniger als $1 \%$ beschränkt. Grundsätzlich sind höhere ${ }^{17} \mathrm{O}$-Konzentrationen auch bei dieser Betriebsweise erzielbar, aber für Kolonnenlängen $\varepsilon S>4$ ist ent-

${ }^{6}$ Vgl. W. Kuhn ${ }^{5}$.

7 M. Thuerkauf, A. Narten u. W. Kuhn, Helv. Chim. Acta 43, 989 [1960]. weder die Menge an erzeugtem ${ }^{17} \mathrm{O}$ praktisch Null oder die Einstellzeit der Kolonne ist für praktische Zwecke zu groß.

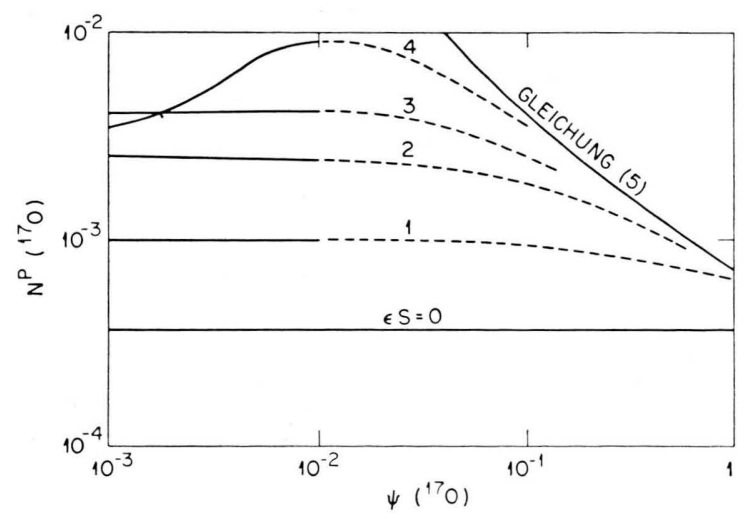

Abb. 4. Anreicherung von Sauerstoff-17 aus dem natürlichen Isotopengemisch in einer Gegenstromkolonne (square cascade) als Funktion des Entnahmeparameters $\psi\left({ }^{17} \mathrm{O}\right)=P / L \varepsilon\left({ }^{17} \mathrm{O}\right)$ für verschiedene Kolonnenlängen $\varepsilon\left({ }^{17} \mathrm{O}\right) S$. (Die gestrichelten Kurventeile wurden von der Operations Analysis Division, ORGDP, durch numerische Integration des Systems (3) gewonnen.)

Um höhere Konzentrationen an Sauerstoff-17 in Millimolmengen zu erzielen, ist es notwendig, Kaskaden aus mehr als einer Kolonne heranzuziehen. Zur Produktion von hochangereichertem ${ }^{17} \mathrm{O}$ ist es sogar erforderlich, Kaskaden aus verschiedenen Trennelementen zu kombinieren. Zur Voranreiche-

8 K. Clusius u. K. Schleich, Helv. Chim. Acta 45, 1702 [1962]. 
rung auf $2-4 \%{ }^{17} \mathrm{O}$ kann eine gestaffelte Wasserdestillationskaskade dienen, deren Produkt elektrolysiert und als Sauerstoffgas einer Thermodiffusionskaskade zur Hochanreicherung zugeführt wird.

Eine solche kombinierte Trennkaskade zur Produktion von Millimolmengen an 50-proz. Sauer- stoff-17 ist seit 1961 im Oak Ridge National Laboratory in Betrieb ${ }^{9}$.

I am indebted to Gordon Czzzer of Metals and Ceramics Division, ORNL, for stimulating discussions, and to Edward von Halle of Operations Analysis Division, ORGDP, for his constructive criticism.

9 A. Narten u. J. S. Drury, in Vorbereitung.

\title{
Messungen der Argon-Diffusion in $\mathrm{KCl}$ und $\mathrm{KBr}$
}

(Edelgasdiffusion in Festkörpern 22)

\author{
A. H. K. Richter und K. E. Zimen \\ Hahn-Meitner-Institut für Kernforschung Berlin \\ (Z. Naturforschg. 20 a, 666-672 [1965]; eingegangen am 2. Februar 1965)
}

\begin{abstract}
Die Diffusion von radioaktivem Argon wurde in reinen und dotierten Einkristallen von $\mathrm{KCl}$ und $\mathrm{KBr}$ sowie in gepreßten Tabletten von $\mathrm{KCl}$ im Temperaturbereich von $300-700{ }^{\circ} \mathrm{C}$ gemessen. Als Aktivierungsenergien wurden gefunden:

unterhalb $400{ }^{\circ} \mathrm{C} \quad 2,4 \mathrm{eV}(55 \mathrm{kcal} / \mathrm{mol})$ für $\mathrm{KCl}$ und $1,5 \mathrm{eV}(36 \mathrm{kcal} / \mathrm{mol})$ für $\mathrm{KBr}$, oberhalb $400{ }^{\circ} \mathrm{C} \quad 0,6 \mathrm{eV}(14 \mathrm{kcal} / \mathrm{mol})$ für $\mathrm{KCl}$ und $0,4 \mathrm{eV}(8 \mathrm{kcal} / \mathrm{mol})$ für $\mathrm{KBr}$.

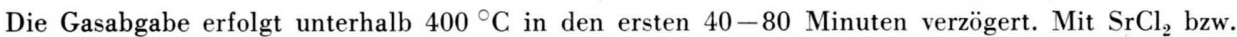
$\mathrm{K}_{2} \mathrm{~S}$ dotierte KCl-Kristalle unterscheiden sich im Diffusionsverhalten nicht von KCl-Einkristallen. In den KCl-Tabletten beeinflussen geschlossene Poren die Edelgasabgabe.
\end{abstract}

Untersuchungen der Edelgasabgabe aus Festkörpern haben praktische Bedeutung für Kernbrennstoffe und allgemeines Interesse für die Erfassung der Struktur und Reaktivität von Festkörpern $\left(Z_{\text {Imen }}{ }^{1}\right)$. Das Diffusionsverhalten von Argon in K-Salzen interessiert einmal als Modellverhalten für Kernbrennstoffe ähnlicher Struktur (Felix, SchmeLING und $Z_{\text {IMEN }}{ }^{2}$ ) sowie im Zusammenhang mit geologischen Altersbestimmungen zwecks Berücksichtigung der Diffusionsverluste (GentNer et al. ${ }^{3,4}$ ). Die vorliegende Arbeit schließt sich an die Untersuchung des Systems KF (Mundt, Richter ${ }^{5}$ ) an.

\section{Experimentelles}

Die meisten Messungen wurden mit einer kontinuierlich arbeitenden Zirkulationsapparatur nach Abb. 1 durchgeführt. Das Durchflußzählrohr hatte eine Massenbelegung von $25-35 \mathrm{mg} / \mathrm{cm}^{2}$ und ein empfindliches Volumen von $70 \mathrm{ml}$ (Volumen des ganzen Gaskreis-

1 K. E. Zimen, Trans. Chalmers Univ. Technol. Göteborg, No. 175 [1956].

2 F. Felix, P. Schmeling u. K. E. Zimen, EUR. 259.d, 1963, p. 13.

3 W. Gentner u. W. Kley, Z. Naturforschg. 10 a, 832 [1955].

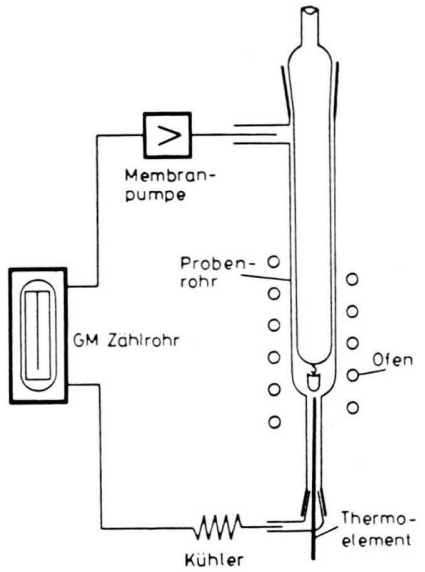

Abb. 1. Schema der kontinuierlich arbeitenden Apparatur mit zirkulierendem Gasstrom.

laufs ca. $300 \mathrm{ml}$ ). Die Membranpumpe (55 Watt) verteilte eine plötzlich eingebrachte Aktivität im Kreislaufsystem in etwa 2 min. Eingebrachte KCl-Einkristalle

4 H. Fechtig, W. Gentner u. S. Kalbitzer, Geochim. Cosmochim. Acta 25, 297 [1961].

5 H. P. Mundt u. A. H. K. Richter, Z. Naturforschg. 20 a, 267 [1965]. 